\title{
Wiggling skyrmion propagation under parametric pumping
}

\author{
H. Y. Yuan, ${ }^{1, *}$ X. S. Wang, ${ }^{2,3}$ Man-Hong Yung, ${ }^{4,5, \dagger}$ and X. R. Wang ${ }^{3,6, \ddagger}$ \\ ${ }^{1}$ Department of Physics, Southern University of Science and Technology, Shenzhen, 518055, Guangdong, China \\ ${ }^{2}$ School of Microelectronics and Solid-State Electronics, \\ University of Electronic Science and Technology of China, Chengdu, Sichuan 610054, China \\ ${ }^{3}$ Physics Department, The Hong Kong University of Science and Technology, Clear Water Bay, Kowloon, Hong Kong \\ ${ }^{4}$ Institute for Quantum Science and Engineering and Department of Physics, \\ South University of Science and Technology of China, Shenzhen, 518055, China \\ ${ }^{5}$ Shenzhen Key Laboratory of Quantum Science and Engineering, Shenzhen, 518055, China \\ ${ }^{6}$ HKUST Shenzhen Research Institute, Shenzhen 518057, China
}

(Dated: August 1, 2018)

\begin{abstract}
We address the problem of how magnetic skyrmions can propagate along a guided direction by parametric pumping. As evidenced by our micromagnetic simulations, skyrmions can hardly be driven by either a static electric field or a static magnetic field alone. Although the magnetic anisotropy can be modified by an electric field, parametric pumping with an oscillating electric field can only excite the breathing modes. On the other hand, a static magnetic field can break rotational symmetry through the Zeeman interaction, but it cannot serve as an energy source for propelling a skyrmion. Here we found that the combination of a perpendicularly oscillating electric field and an in-plane static magnetic field can drive a skyrmion undergoing a wiggling motion along a well defined trajectory. The most efficient driving occurs when the frequency of the oscillating field is close to that of the breathing motion. The physics is revealed in a generalized Thiele equation where a net spin current excited by the parametric pumping can drive the skyrmion propagation through angular momentum transfer. Compared with other alternative proposals, our results open new possibilities for manipulating skyrmions in both metals and insulators with low-power consumption. The oscillating skyrmion motion can also be a microwave generator for future spintronic applications such as an nano-tool on a diamond Nitrogen-Vacancy center.
\end{abstract}

\section{INTRODUCTION}

Magnetic skyrmions are topological structures that were observed in a class of magnetic materials with broken inversion symmetry ${ }^{1-8}$. In comparison with magnetic bubbles ${ }^{9}$ and domain walls ${ }^{10}$, skyrmions are relatively small $(1-100 \mathrm{~nm})^{11}$, and can be driven with a lower current density $\left(10^{6} \mathrm{~A} / \mathrm{m}^{2}\right)^{12}$, making them ideal for being information carriers. Recently, various methods have been proposed for controlling skyrmion motion, including electric currents ${ }^{6,12-14}$, spin waves ${ }^{15,16}$, microwaves ${ }^{17,18}$, and temperature gradient ${ }^{19-21}$. In particular, skyrmions in a metal driven by an electric current can move both parallel and transverse to the current, known as the skyrmion Hall effect ${ }^{14,22}$. However, a current does not work for insulating materials that may have lower damping, lower power consumption, and better controllability. To manipulate skyrmions in insulators, temperature gradient is proposed as a control knob through the spin transfer torque. Unfortunately, similar to the magnonic spin transfer torque induced domain-wall motion, ${ }^{23}$, the effectiveness of thermal magnons remains a problem in practice. Thus, finding new control knobs for skyrmions is an interesting issue in spintronics.

Parametric pumping refers to a parameter cycling or oscillation that can result in a net charge/spin transport. The system response to a parametric pumping may be strong (at resonance) if the parameter cycling frequency matches with the system intrinsic frequency. In recent years, using electric fields to manipulate mag- netic states is a focus in nanomagnetism ${ }^{24-35}$, because of its high controllability and low energy consumption. Electric field can modify material parameters such as exchange stiffness ${ }^{26,27}$, anisotropy coefficient ${ }^{29-31}$, and even the strength of the Dzyaloshinskii-Moriya interaction (DMI) ${ }^{28,36,37}$. However, the behavior of a skyrmion subject to a parametric pumping remains a largelyunexplored topic.

A perpendicularly-oscillating electric field (POEF) on a vertically-magnetized film can periodically modify the magnetic anisotropy ${ }^{29-31}$, resulting in a parametric pumping. However, a skyrmion in such a film undergoes only a breathing motion, instead of propagating along a well-defined direction. In this paper, we show that a POEF together with an in-plane static magnetic field, which breaks skyrmion rotational symmetry, can drive a skyrmion to move along a given direction. The motion is attributed to the spin current that transfers its angular momentum to the skyrmion wall and is associated with skyrmion breathing motion. The skyrmion velocity reaches its maximum when the POEF frequency matches that of the breathing motion. These results are numerically verified by micromagnetic simulations and are analytically justified from the generalized Thiele equation.

\section{MODEL AND METHODS}

We consider a perpendicularly-magnetized film with a skyrmion in the center as shown in Fig. 1a. The skyrmion is stabilized by the competition between ex- 
change interaction, anisotropy and interface $\mathrm{DMI}^{36,37}$ from the asymmetric interfaces of magnetic and nonmagnetic layers. The skyrmion dynamics is governed by the Landau-Lifshitz-Gilbert (LLG) equation,

$$
\frac{\partial \mathbf{m}}{\partial t}=-\gamma \mathbf{m} \times \mathbf{H}_{\mathrm{eff}}+\alpha \mathbf{m} \times \frac{\partial \mathbf{m}}{\partial t},
$$

where $\mathbf{m}, \gamma, \alpha$ are respectively the unit vector of the magnetization, gyromagnetic ratio, and the Gilbert damping. $\mathbf{H}_{\mathrm{eff}}=2 A \nabla^{2} \mathbf{m}+2 K_{u} m_{z} \mathbf{e}_{z}+\mathbf{H}_{d}+H \mathbf{e}_{y}+\mathbf{H}_{\mathrm{DM}}+\mathbf{H}_{\mathrm{so}}$ is the effective field including the exchange field, crystalline anisotropy field, dipolar field $\mathbf{H}_{d}$, external field $H \mathbf{e}_{y}$ along the y-direction, DMI field $\mathbf{H}_{\mathrm{DM}}$ and spinorbit field ${ }^{38} \mathbf{H}_{\text {so }}$ due to the electric field. $A$ is the exchange stiffness and $K_{u}$ is the anisotropy coefficient. The spin-orbit field is induced by the applied electric field through spin-orbit interaction and can be divided into the damping-like components and field-like components ${ }^{38}$, i.e. $\mathbf{H}_{\mathrm{so}}=\zeta_{D} E m_{z} \mathbf{m} \times \mathbf{e}_{z}+\zeta_{F} E m_{z} \mathbf{e}_{z}$, here $E$ is the electric field while $\zeta_{D}$ and $\zeta_{F}$ are the torque conversion coefficients. To investigate the skyrmion structure and its dynamics in an electric field, we use the Mumax3 package ${ }^{39}$ to numerically solve the LLG equation. The film size is of $128 \mathrm{~nm} \times 128 \mathrm{~nm} \times 0.4 \mathrm{~nm}$, if it is not stated otherwise. The model parameters are $A=10 \times 10^{-12} \mathrm{~J} / \mathrm{m}, D=0.003 \mathrm{~J} / \mathrm{m}^{2}, M_{s}=9.2 \times$ $10^{5} \mathrm{~A} / \mathrm{m}, K_{u}=1.157 \times 10^{6} \mathrm{~J} / \mathrm{m}^{3}, \zeta_{F}=0.02 \mathrm{~J} /\left(\mathrm{V} \cdot \mathrm{m}^{2}\right)$ to mimic $\mathrm{CoPd}^{38}$. The Gilbert damping varies from 0.02 to 0.2 . We focus on the influence of field-like spin-orbit torque on skyrmion dynamics and take $\zeta_{D}=0$ in the simulations.

\section{RESULTS}

\section{A. Skyrmion structures}

Let us first look at the skyrmion structures under a static electric field $(E)$. Figure $1 \mathrm{~b}$ shows that the skyrmion size $R_{s}$ decreases with the increase of electric field with a typical skyrmion structure shown in the left inset for $E=0$ and $H=0$. The skyrmion size can be described by ${ }^{40}$

$$
R_{s}=\pi D \sqrt{\frac{A}{16 A K_{\mathrm{eff}}^{2}-\pi^{2} D^{2} K_{\mathrm{eff}}}},
$$

where $K_{\text {eff }}=K_{u}+\zeta_{F} E / 2-\mu_{0} M_{s}^{2} / 2$. Here the long-range dipolar interaction is approximated as the shape anisotropy $\mu_{0} M_{s}^{2} / 2$ along the $z$-axis, which is well justified for a magnetic thin film. This is of the variational ${ }^{40-42}$ result obtained by assuming the skyrmion profile along radial direction as a $360^{\circ}$ domain wall with skyrmion size and skyrmion wall width as two optimization parameters. The red solid line in Fig. 1b is Eq. (2) that describes well the simulation results (circles) for $E>-3.6 \times 10^{6} \mathrm{~V} / \mathrm{m}$. For electric fields
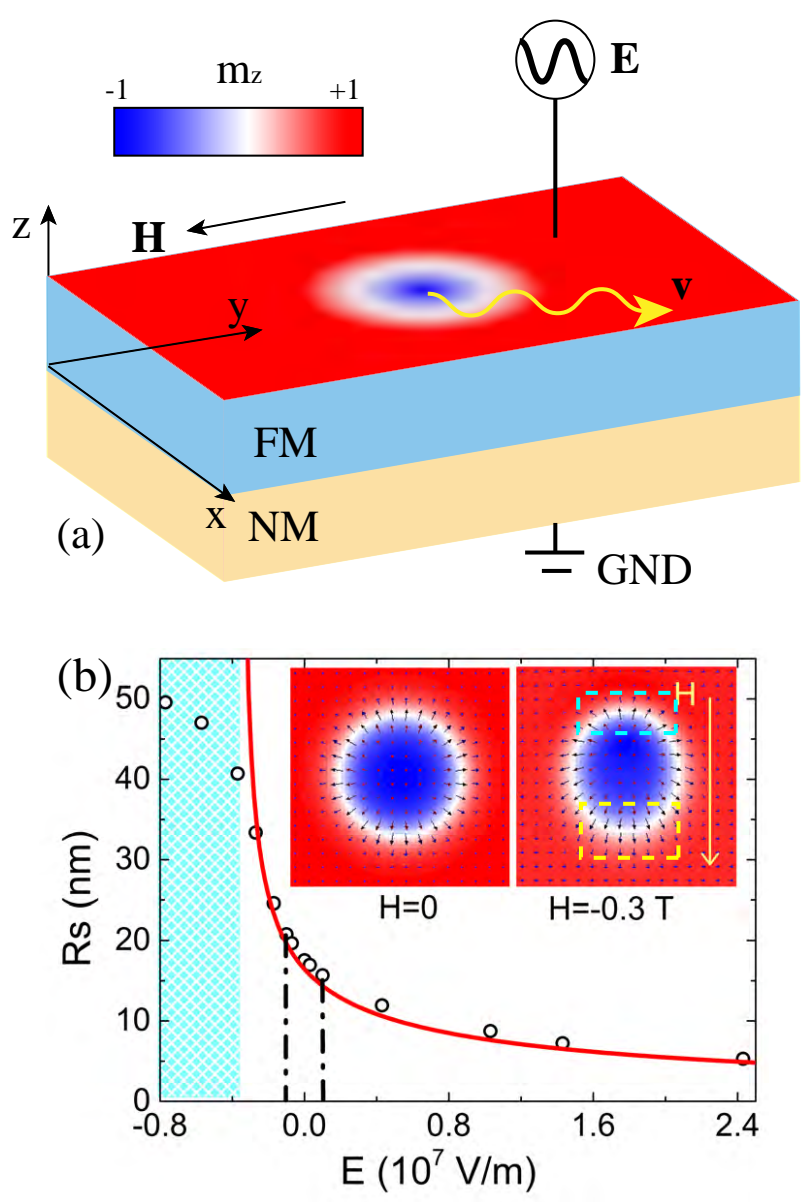

FIG. 1. (color online) (a) Schematic illustration of a magnetic layered structures. The colors encode $m_{z}$ value with the color bar shown in the top-left panel. A magnetic field along the y direction breaks the rotational symmetry of skyrmions with respect to $z$-axis. An oscillating electric field is applied normally ( $z$-direction) to the film. The skyrmion moves wiggly in the $x y$-plane as illustrated by the yellow arrow. (b) Skyrimion radius as a function of electric field in the absence of magnetic fields. The blue shadowed region below $-3.6 \times 10^{6} \mathrm{~V} / \mathrm{m}$ is for the unstable skyrmions. The red line is Eq. (2). The insets show the symmetric and asymmetric skyrmion structures for $H=0$ (left) and $-0.3 \mathrm{~T}$ (right), respectively. The dashed lines indicate the field variation range within which $R_{s}$ varies from $20.8 \mathrm{~nm}$ to $15.7 \mathrm{~nm}$.

smaller than that value, the skyrmion size (diameter $>80$ $\mathrm{nm})$ is comparable with the system size $(128 \mathrm{~nm})$ and the boundary effect becomes pronounced. In an infinite film, the skyrmion should proliferate and becomes unstable $^{41}$ at the critical field $E_{c}=\pi^{2} D^{2} /\left(8 A \zeta_{F}\right)-$ $\left(2 K_{u}-\mu_{0} M_{s}^{2}\right) / \zeta_{F}=-3.6 \times 10^{6} \mathrm{~V} / \mathrm{m}$ (blue shadowed region). Under an in-plane field, the skyrmion deforms and elongates along the field direction as shown in the right inset of Fig. 1b for $H=-0.3 \mathrm{~T}$. Here the top and bottom skyrmion walls become thinner and thicker, respectively, to take the advantage of the Zeeman effect. The larger the in-plane field, the larger the width differ- 

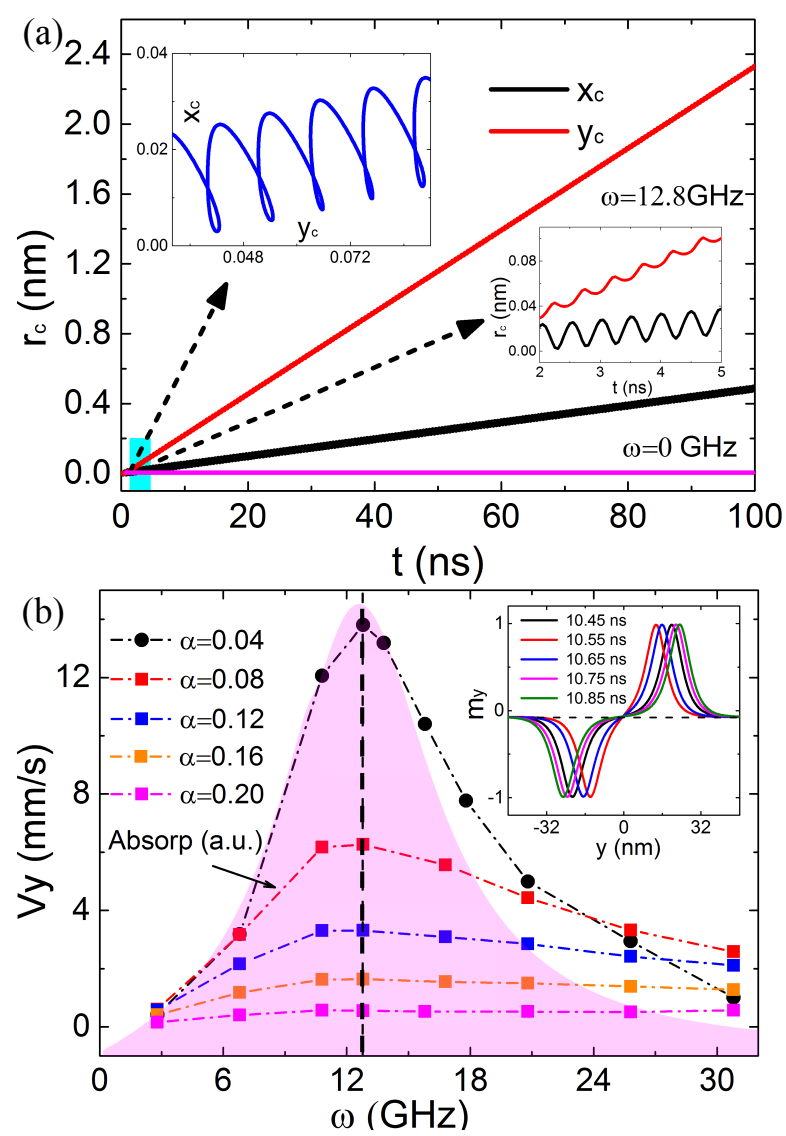

FIG. 2. (color online) (a) Evolution of skyrmion center $\mathbf{r}_{c}=\left(x_{c}, y_{c}\right)$ for $\omega=12.8 \mathrm{GHz}$ and $\omega=0$, respectively. The slope of the $x_{c}-t$ and $y_{c}-t$ curves give the skyrmion velocity $v_{x}$ and $v_{y}$, respectively. The right inset is the zoomin plots of $x_{c}$ (black) and $y_{c}$ (red) plots that show a wiggling skyrmion motion with a wiggling trajectory shown in the left inset. (b) Average skyrmion velocity as a function of electric field frequency for $\alpha=0.04,0.08,0.12,0.15$ and 0.20 , respectively. The pink shadow illustrates the absorption spectrum. The inset is the snapshots of distribution of $m_{y}$ along $x=0$ at different times. The extremes locate the skyrmion wall centers. The oscillations of wall centers with time demonstrate a breathing motion of the skyrmion under parametric pumping.

ence between the top and the bottom skyrmion walls is (See the Appendix A).

\section{B. Skyrmion motion}

To describe the skyrmion motion of the asymmetric skyrmions under a harmonic POEF of $\mathbf{E}=E_{0} \sin (\omega t) \mathbf{e}_{z}$ and an in-plane magnetic field, we define the skyrmion position as topological charge weighted center ${ }^{19}: \mathbf{r}_{c} \equiv$ $1 /(4 \pi Q) \int \mathbf{m} \cdot\left(\partial_{x} \mathbf{m} \times \partial_{y} \mathbf{m}\right) \mathbf{r} d S$ with $Q \equiv 1 /\left(4 \pi \int \mathbf{m} \cdot\right.$ $\left(\partial_{x} \mathbf{m} \times \partial_{y} \mathbf{m}\right) d S$ being the skyrmion number. Figure $2 \mathrm{a}$ shows the time dependence of skyrmion position for $\omega=$ 0 and $12.8 \mathrm{GHz}$, respectively. When a static electric field is applied, i.e. $\omega=0$, the skyrmion does not move. When $\omega=12.8 \mathrm{GHz}$, the skyrmion shows a wiggling motion in both the $x$ - and the $y$-directions with typical trajectories shown in the insets of Fig. 2a. Figure 2b is the fieldfrequency-dependence of the average skyrmion velocity along the y-direction $\left(v_{y}\right)$ for various damping coefficients ranging from 0.04 to $0.20 . v_{y}$ is peaked around $12.8 \mathrm{GHz}$, almost independent of $\alpha$.

In order to check whether the peak is associated with the parametric resonance that occurs when the POEF frequency matches with a skyrmion intrinsic frequency, we consider the dynamical susceptibility of the system to a sine field of $E_{z}(t)=E_{0} \sin \omega t /(\omega t)$, defined as $\left\langle m_{z}(t)\right\rangle=\chi_{z z}(\omega) E_{z}(t)$, where $\left\langle m_{z}(t)\right\rangle$ is the average $m_{z}$. The energy absorption of the system, proportional to $\operatorname{Im}(\chi(\omega))^{43}$, is shown by the pink shadowed region in Fig. 2b. The absorption peak is located around 13 $\mathrm{GHz}$ that coincides with the maximal skyrmion velocity. The skyrmion response to the POEF of $\omega=12.8 \mathrm{GHz}$ is shown in the inset of Fig. 2b that plots the snapshots of $m_{y}$ along $x=0$. The positions with extreme $m_{y}$ values are the skyrmion wall centers. The center positions oscillate back-and-forth with time. This shows clearly a strong breathing motion of the skyrmion ${ }^{44-46}$. Thus, the velocity peak corresponds to resonance of the POEF with the skyrmion breathing mode of $13 \mathrm{GHz}$. It should note a minor peak around $10.4 \mathrm{GHz}$ that will influences the skyrmion velocity for $\alpha<0.01$ (See the Appendix B).

\section{Generalized Thiele equation}

The wiggling motion of skyrmion center shown in Fig. $2 \mathrm{a}$ accompanies the skyrmion breathing. The breathing motion emits spin waves (a magnon spin current) similar to the spin wave emission by domain wall motion ${ }^{47}$. The emitted spin waves across the skyrmion wall, transfer the angular momentum to a skyrmion and drive the skyrmion to move, similar to spin transfer torque induced domain wall motion. Because the rotational symmetry of the skyrmion is broken by the in-plane field, the magnon current should have different components along the field direction (+y-direction) and the $x$-direction. To understand the behavior, we consider the generalized Thiele equation $^{12}$ (See the Appendix C),

$$
\mathbf{G} \times\left(\mathbf{v}-\mathbf{j}^{(m)}\right)+\mathbf{D} \cdot\left(\alpha \mathbf{v}-\beta \mathbf{j}^{(m)}\right)=0
$$

where $\mathbf{G}=G \mathbf{e}_{z}=4 \pi Q \mathbf{e}_{z}$ is the skyrmion gyrovector proportional to the skyrmion number $Q$, and $D_{i j}=$ $\int \partial_{i} \mathbf{m} \cdot \partial_{j} \mathbf{m} d S$ is the dissipation tensor. $\mathbf{v}=\left(v_{x}, v_{y}\right)$ is average skyrmion velocity, and $\beta$ describes the misalignment of magnon polarization and local magnetization that is zero here. $\mathbf{j}^{(m)}$ is the average magnon current. The skyrmion velocity can be obtained from Eq. (8)

$$
v_{x}=\frac{j_{x}^{(m)}-\alpha \kappa j_{y}^{(m)}}{1+\alpha^{2} \kappa^{2}}, v_{y}=\frac{j_{y}^{(m)}+\alpha \kappa j_{x}^{(m)}}{1+\alpha^{2} \kappa^{2}}
$$

where $\kappa=D_{x x} / G$. Figure 3a shows that $v_{y}$ decreases with the damping hyperbolically while $v_{x}$ is almost a 

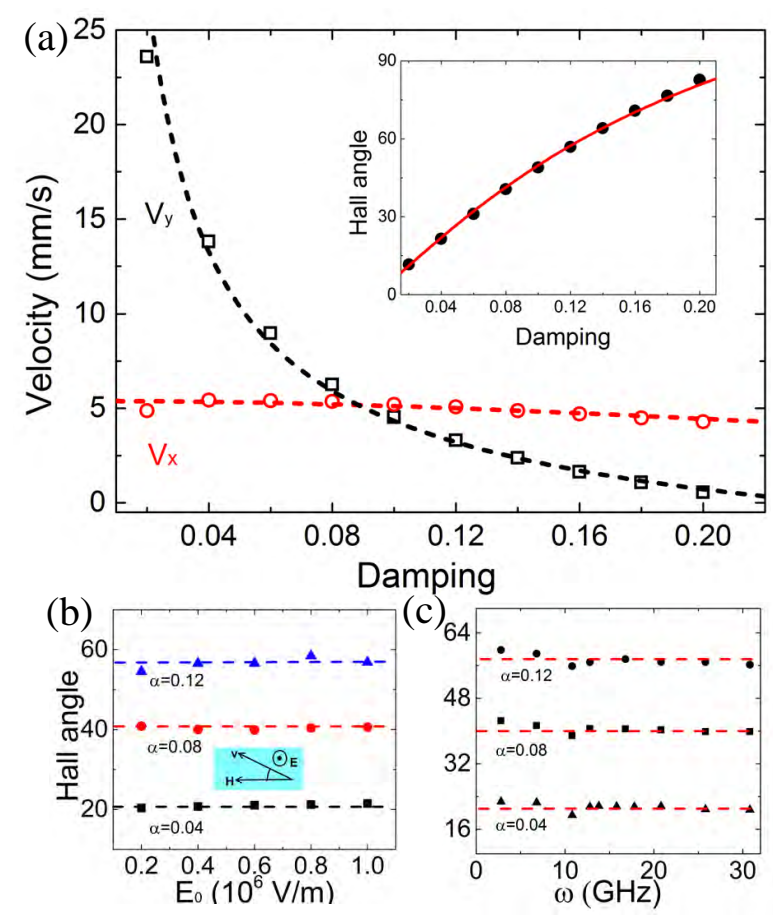

FIG. 3. (color online) (a) Average skyrmion velocity as a function of damping at $\omega=12.8 \mathrm{GHz}$. The symbols are simulation data, and the dashed lines are the solutions of the Thiele equation. The inset shows the Hall angle defined as $\arctan \left(v_{x} / v_{y}\right)$ as a function of damping parameter. The solid line is theoretical calculations. (b) and (c) Electric field strength and field frequency dependence of Hall angle under different dampings. The symbols are simulation data, and the horizontal dashed lines are used to guide eyes.

constant, which suggests that the magnon current $j_{y}^{(m)}$ is inversely proportional to $\alpha$ while $j_{x}^{(m)}$ is damping independent since $\alpha \kappa \ll 1$ in Eq. (4), i.e. $j_{y}^{(m)}=$ $C_{y} / \alpha, j_{x}^{(m)}=C_{x}$. Using the parameters $C_{x}=2 \times 10^{-5}$, $C_{y}=2.7 \times 10^{-6}$, Eq. (4) can indeed fit the numerical data (symbols) perfectly as shown in Fig. 3a. Furthermore, the skyrmion Hall angle defined as $\operatorname{atan}\left(v_{x} / v_{y}\right)=$ $\operatorname{atan}\left(\left(j_{x}^{(m)}-\alpha \kappa j_{y}^{(m)}\right) /\left(j_{y}^{(m)}+\alpha \kappa j_{x}^{(m)}\right)\right)$ is calculated and plotted as the red line in the inset of of Fig. 3a. Again, it perfectly describes the numerical results (circles). Interestingly, at given $\alpha$, the Hall angle is insensitive to both the amplitude and frequency of electric field, as shown in Fig. $3 \mathrm{~b}$ and c.

\section{Skyrmion inflation and deflation}

The skyrmion size under an POEF oscillates periodically because the electric field modifies the magnetic anisotropy. According to Eq. (2), the skyrmion size should vary in the range of $R_{\min }=R_{s}\left(E=E_{0}\right)$ and $R_{\max }=R_{s}\left(E=-E_{0}\right)$ as shown in the cyan rectangle in Fig. 4a. However, micromagnetic simulations show
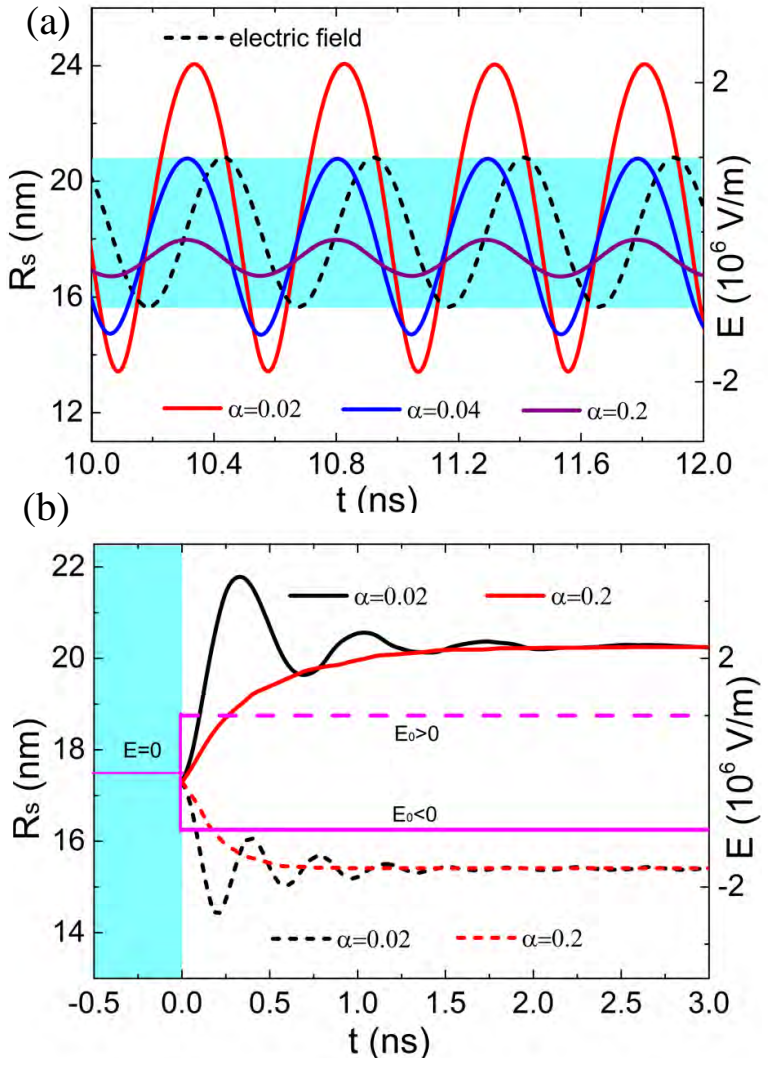

FIG. 4. (color online) (a) Skyrmion size as a function of time at $\omega=12.8 \mathrm{GHz}$ for $\alpha=0.02$ (red line), 0.04 (blue line), and 0.20 (purple line), respectively. The cyan rectangle is the region of size fluctuation by taking static field $E \in$ $\left[E_{0},-E_{0}\right]$. The dashed line is the oscillation electric field. (b) Time dependence of skyrmion size after a sudden quench of anisotropy by using a step electric field switched on at $t=0 \mathrm{~ns}$ for $\alpha=0.02$ (black line) and and 0.2 (red line). The solid and dashed lines are for $E_{0}<0$ (dashed horizontal line) and $E_{0}>0$ (solid horizontal line), respectively.

that the skyrmion size oscillates out of this range for $\alpha \leq 0.04$ and falls into this range for $\alpha>0.04$, as shown in Fig. 4a. This indicates that skyrmion under parametric pumping has an inertia. The steady response of the skyrmion size to an applied harmonic POEF (dashed lines) is shown in Fig. 4a for $\alpha=0.002$ (red), 0.04 (blue) and 0.2 (purple), respectively. They showed the typical breathing motion (expansion-and-contraction). Evidently, the skyrmion size variation has a phase lag to its driving POEF. The lagged phase increases with the damping, similar to a damped harmonic oscillator ${ }^{48}$. It is under damped for a lower $\alpha(\leq 0.04)$ so that the stored energy from POEF will push the skyrmion to expand beyond its static size. It is over-damped for a larger $\alpha$. The skyrmion motion lags behind the external pumping field so much that the skyrmion cannot reach its maximal or minimal sizes corresponding to the minimal and maximal effective anisotropies. To further substantiate the damping dependence of skyrmion size oscillation, we 
consider how the skyrmion size responds to a sudden switching of a constant electric field. The results are shown in Fig. 4b. The solid (dashed) lines are the evolution of skyrmion size $R_{s}$ to a constant electric field $E=1.0 \times 10^{6} \mathrm{~V} / \mathrm{m}\left(E=-1.0 \times 10^{6} \mathrm{~V} / \mathrm{m}\right)$ switched on at $t=0$ for $\alpha=0.02$ (black) and $\alpha=0.2$ (red), respectively. The skyrmion size oscillates on its way to the equilibrium value for $\alpha=0.02$ while it takes a long time for $R_{s}$ to monotonically relax to its equilibrium value for $\alpha=0.2$. Take $E<0$ as an example, the intermediate skyrmion size can be larger than the equilibrium value for $\alpha=0.02$ while it is always smaller than the equilibrium value for $\alpha=0.2$. For a fast oscillating electric field, the skyrmions size can be kept at intermediate values periodically for small damping since the skyrmion cannot dissipate its energy timely. This explains the observation of extraordinarily large/small skyrmion in Fig. 4a for small damping.

\section{E. Spin qubit manipulation}

The dipolar field outside the magnetic film will oscillate periodically accompanying the skyrmion size oscillates under parametric pumping. Since the oscillation frequency of the dipolar field can reach $\mathrm{GHz}$ level as shown in Fig. 2, the oscillating skyrmion can be a microwave generator useful for spin qubit manipulation in quantum information science.

Figure 5a shows a FM/NM bilayer with a nanodiamond placed on top of the FM layer. The spin qubit inside the Nitrogen-Vacancy (NV) center of the diamond interacts with the skyrmion via the dipoalr interaction. To see how the qubit in a NV center $(S=1)$ responds to the oscillating dipolar field, we recall the Hamiltonian of a NV center, ${ }^{49}$

$$
H_{N V}=D_{0}\left(S_{z^{\prime}}\right)^{2}+\gamma_{N V} S_{x^{\prime}}\left(B_{x^{\prime}}^{s k x}+B_{x^{\prime}}\right)+\gamma_{N V} S_{z^{\prime}} B_{z^{\prime}},
$$

where the $z^{\prime}$-axis is chosen to align along one of the NV symmetry axes and the $x^{\prime}$-axis is along the magnetic film normal direction (z-axis). $S_{x^{\prime}}, S_{y^{\prime}}$, and $S_{z^{\prime}}$ are the spin1 operators along the $x^{\prime}, y^{\prime}, z^{\prime}$ directions, $D_{0} / 2 \pi=2.87$ $\mathrm{GHz}$ is the zero-field splitting, $\gamma_{N V} / 2 \pi=2.8 \mathrm{MHz} / \mathrm{Oe}$ is the gyromagnetic ratio of nitrogen atom. Here $B_{x^{\prime}}$ is a bias field to cancel the direct part in $B_{x^{\prime}}^{s k x}$. We have neglected the magnetic field generated by the oscillating electric field, which can be estimated by solving the Maxwell equation as $L \omega E_{0} /\left(4 c^{2}\right) \sim 10^{-9} \mathrm{~T}$, where $L=128 \mathrm{~nm}$ is the film size, $\omega$ and $E_{0}$ are the frequency and amplitude of the electric field, respectively, and $c$ is the speed of light.

Without external fields $\left(B_{z^{\prime}}\right)$, the ground state of a NV center is $m_{s}=0$ while the two excited states $m_{s}= \pm 1$ are degenerate in energy. With a static magnetic field, the degeneracy of $m_{s}= \pm 1$ is broken, which results in a three level system of $m_{s}=0,-1,+1$, respectively, as shown in
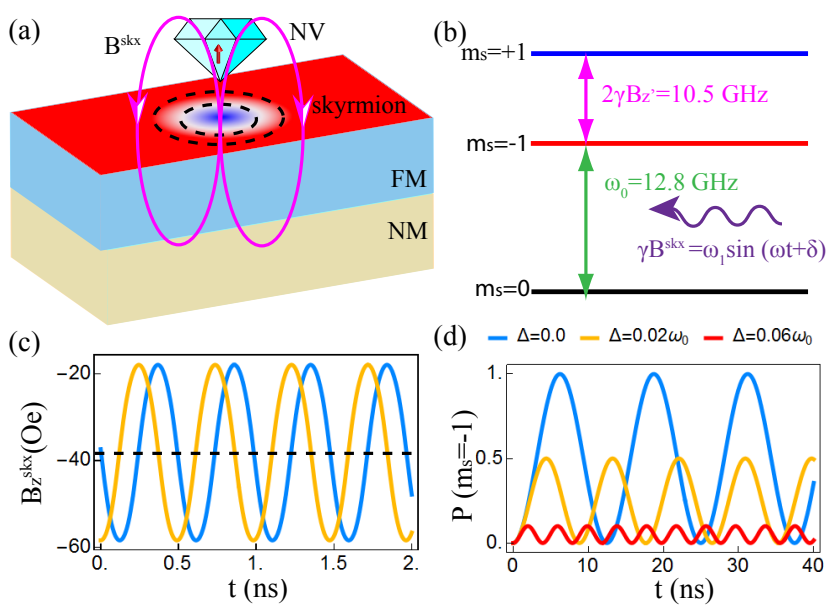

(d) $-\Delta=0.0-\Delta=0.02 \omega_{0}-\Delta=0.06 \omega_{0}$

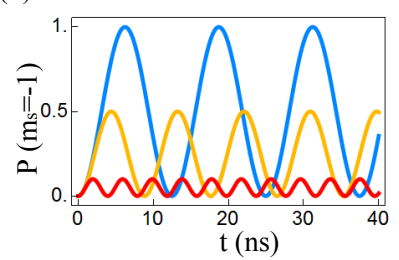

FIG. 5. (color online) (a) Schematic illustration of a FM/NM bilayer with a nanodiamond placed on top of the FM layer. The spin qubit in the NV center of the diamond interacts with the skyrmion via the dipolar field $\mathbf{B}^{\text {skx }}$ generated by the oscillating skyrmion (pink lines). (b) Schematic illustration of the energy levels of the ground state of a NV center under a Zeeman field $B_{z^{\prime}}=298$ Oe. (c) The oscillation of the dipolar fields at $d=16 \mathrm{~nm}$ with a phase shift $\pi / 2$. (d) Population rate of the excited state $\left(m_{s}=-1\right)$ as a function of time for detuning $\Delta=0$ (blue line), $0.02 \omega_{0}$ (orange line), and $0.06 \omega_{0}$ (red line), respectively.

Fig. 5b. Here we use a static magnetic field to tune the splittng of $m_{s}= \pm 1$ such that the energy gap between $m_{s}=0$ and $m_{s}=-1$ is close to the frequency of oscillating dipolar field generated by the breathing skyrmion ( $\sim 12.8 \mathrm{GHz}$ for our parameters). Moreover, we notice that energy shift induced by the dipolar field is much smaller than the energy gap $\left(\omega_{1} \ll \omega_{0}, \omega_{1} \ll 2 \gamma B_{z^{\prime}}\right)$, then the transition between the energy levels $m_{s}=0$ and $m_{s}=-1$ dominates the absorption process and the three level system can be reduced to a well-studied two level system. By initializing the NV center to $m_{s}=0$ and turning on the breathing motion of skyrmions, the population rate of $P\left(m_{s}=-1\right)$ will evolve from the initial state $(P=0)$, according to the well-known Rabi formula $^{50} P\left(m_{s}=-1\right)=|C(t)|^{2}=\omega_{1}^{2} / \Omega^{2} \sin ^{2}(\Omega t / 2)$, where $\Omega=\sqrt{\left(\omega_{0}-\omega\right)^{2}+\omega_{1}^{2}}$ is the Rabi frequency, $\omega_{0}=D_{0}-\gamma_{N V} B_{z^{\prime}}, \omega_{1}=\gamma_{N V} B_{x^{\prime}}^{s k x} / \sqrt{2}, B_{x^{\prime}}^{s k x}$ is the oscillation amplitude of the dipolar field, and $\Delta=\omega_{0}-\omega$ is defined as the detuning of dipolar field from the resonant frequency,

Figure 5c shows a typical oscillation of the dipolar field generated by a breathing skyrmion and Figure. $5 \mathrm{~d}$ shows the oscillation of the population rate of $m_{s}=-1$ under this dipolar field for detuning $\Delta=0$ (blue line), $0.02 \omega_{0}$ (orange line), and $0.06 \omega_{0}$ (red line), respectively. At the resonant condition $(\Delta=0)$, the population rate oscillates between 0 and 1 periodically with the frequency that is equal to the oscillation amplitude of the dipolar field $\omega_{1}=252 \mathrm{MHz}$, which is a typical Rabi signal. As the detuning increases to $\Delta=0.02 \omega_{0}$, the maximum 
population of the excited state is near 0.5. As the detuning increases further, the population of the excited state keeps decreasing and finally approaches zero.

\section{DISCUSSIONS AND CONCLUSIONS}

In conclusion, the combination of parametric pumping by a POEF and an in-plane static magnetic field can drive skyrmions undergoing a wiggling motion. The skyrmion velocity reaches its maximum value when the POEF frequency matches with the skyrmion breathing frequency. Our results show a promising avenue for manipulating skyrmions motion in both metallic and insulating magnetic materials. Moreover, the role of in-plane field may be replaced by the exchange bias field in a FM/Antiferromagnet bilayer such that all electric control of skyrmion dynamics can be realized.

Remarkably, temperature-gradient driven skyrmions exhibit a similar damping dependence of the skyrmion velocity as those reported here by parametric pumping. Specifically, the longitudinal (field-direction) velocity quickly decreases with damping while the transverse velocity is insensitive to the damping ${ }^{19}$. The skyrmion velocity under the two driven forces are at the same order of $\mathrm{cm} / \mathrm{s}^{19,20}$. These coincidence may be attributed to the fact that both the electric field and thermal driven skyrmion motion originate from non-uniform magnon flow. Moreover, the skyrmion Hall angle induced by parametric pumping is insensitive to both pumping frequency and pumping amplitude as shown in Fig. 3b and c. This feature is desirable in manipulating skyrmion trajectory in practice.

Although our simulations focus on the Néel skyrmions, the physics should be applicable to Bloch skyrmions (See the Appendix D). Moreover, parametric pumping can also be realized through the cycling of the exchange stiffness and DMI strength besides of the anisotropy studied here. One should expect similar behavior of the skyrmion motion as that in Fig. 1a (See the Appendix E) when other parameter cycling is used. In this sense, parametric pumping is a universal control knob for skyrmion motion. As a comparison, the combined interaction of microwave field and an in-plane field could drive a skyrmion to move in a straight line without any wiggling ${ }^{17}$. The Hall angle dramatically depends on the in-plane field as well as the microwave frequency, which is very different from our observation shown in Fig. 3bc. The anomalous skyrmion size oscillation shown in Fig. 4 was not found in those publications.

\section{ACKNOWLEDGMENTS}

HYY acknowledges the help communication with Weiwei Wang. This work was financially supported by National Natural Science Foundation of China (Grants No. 61704071), Natural Science Foundation of Guang- dong Province (2017B030308003) and the Guangdong Innovative and Entrepreneurial Research Team Program (No. 2016ZT06D348), and the Science Technology and Innovation Commission of Shenzhen Municipality (ZDSYS20170303165926217, JCYJ20170412152620376). XRW was supported by the NSFC Grant (No. 11774296) as well as Hong Kong RGC Grants (Nos. 16301518 and 16301816).

\section{APPENDIX A: IN-PLANE FIELD DEPENDENCE OF SKYRMION PROFILE}

Figure 6a shows the spin configurations as the in-plane field decreases from $0 \mathrm{~T}$ to $-1.0 \mathrm{~T}$. For $|H|<0.9 \mathrm{~T}$, the skyrmion deforms more and more severely with the increase of fields and finally becomes unstable for $|H| \geq 0.9$ $\mathrm{T}$. The skyrmion size first decreases and then increases slightly with the field as shown in Fig. 6c. To see the asymmetric deformation clearly, a typical spin distribution in the $\mathrm{y}$ direction is plotted in Fig. 6b. Here the skyrmion wall with spins parallel to the field expands (cyan region) while the skyrmion wall with anti-parallel orientations with the fields shrinks (pink region).

\section{APPENDIX B: GENERALIZED THIELE EQUATION}

To derive the generalized Thiele equation that describes the drift motion of skyrmion center, we start from the Landau-Lifshitz-Gilbert (LLG) equation that governs the dynamic precessions of the spins inside a skyrmion, i.e.,

$$
\frac{\partial \mathbf{m}}{\partial t}=-\gamma \mathbf{m} \times \mathbf{H}_{\mathrm{eff}}+\alpha \mathbf{m} \times \frac{\partial \mathbf{m}}{\partial t} .
$$

where $\mathbf{m}$ is the normalized magnetization, $\gamma$ is gyromagnetic ratio, $\alpha$ is Gilbert damping that represents the energy dissipation rate of the system, $\mathbf{H}_{\text {eff }}$ is the effective field acting on the magnetization. To distinguish the drifting of skyrmion position and the oscillation of skyrmion size, we decompose the magnetization motion into a slow motion mode $\left(\mathbf{m}_{s}\right)$ and a fast motion mode $\left(\mathbf{m}_{\mathrm{f}}\right)$, i.e. $\mathbf{m}=\left(1-\mathbf{m}_{f}^{2}\right) \mathbf{m}_{s}+\mathbf{m}_{\mathrm{f}}$, where the slow mode represents the equilibrium configuration evolution of the skyrmions while the fast mode refers to the spin wave excitation around the equilibrium configuration of skyrmion. Substituting the decomposition back into the LLG equation (6) and taking a long time (many oscillation periods of the fast mode) average, the dynamic equation of the slow mode can be written as ${ }^{19}$

$$
\frac{\partial \mathbf{m}_{s}}{\partial t}=-\gamma\left\langle\mathbf{m}_{\mathrm{f}} \times \mathbf{H}_{\mathrm{f}}\right\rangle+\alpha \mathbf{m}_{s} \times \frac{\partial \mathbf{m}_{s}}{\partial t} .
$$

where $\mathbf{H}_{\mathrm{f}}$ is the revised effective field $\mathbf{H}_{\mathrm{eff}}(\mathbf{m})$ with $\mathbf{m}$ replaced by $\mathbf{m}_{\mathrm{f}}$. Since $\mathbf{m}_{\mathrm{f}}$ represents the magnon excitation around equilibrium configuration, $\left\langle\mathbf{m}_{\mathrm{f}} \times \mathbf{H}_{\mathrm{f}}\right\rangle$ can 

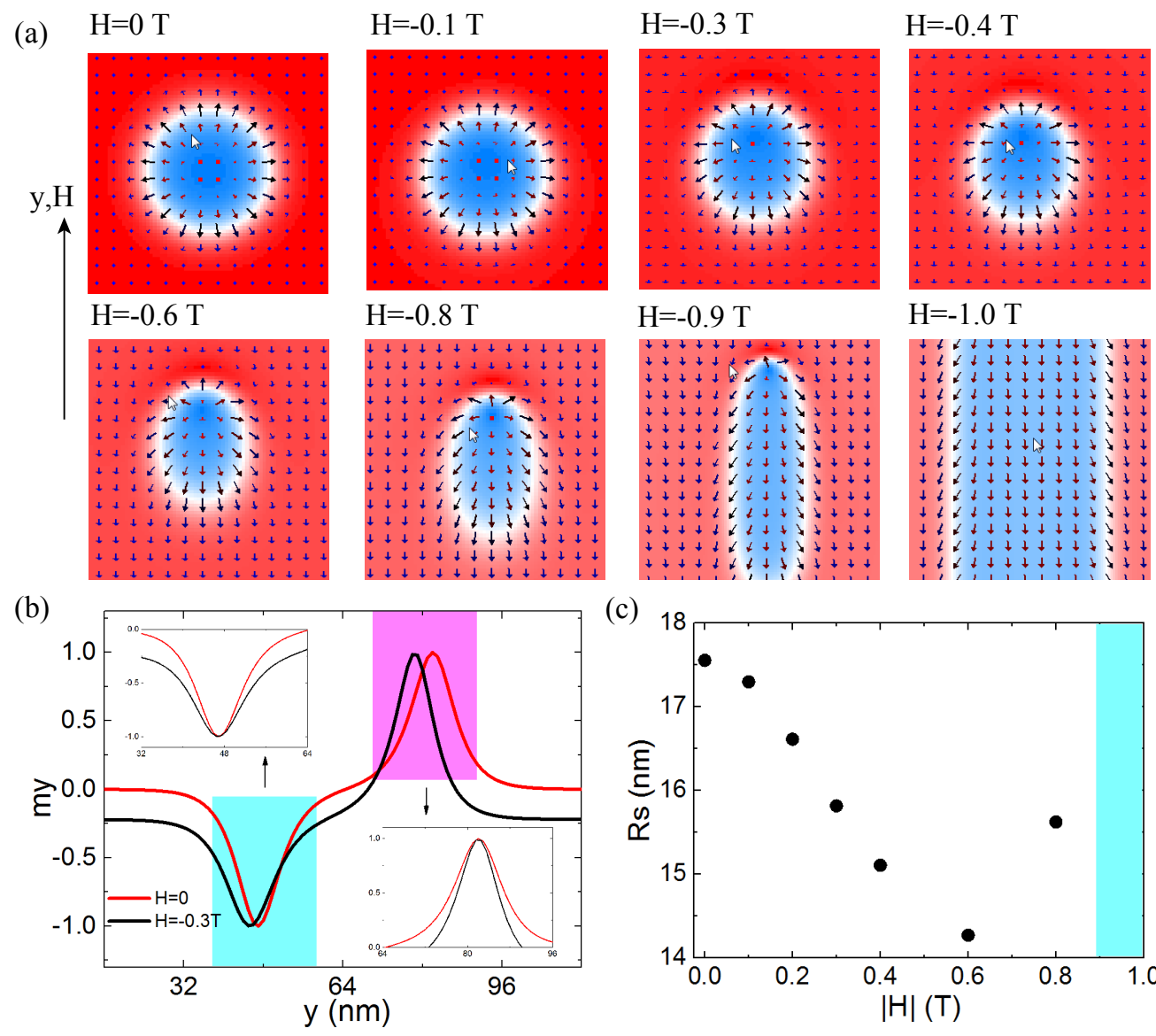

$\mathrm{H}=-0.9 \mathrm{~T}$

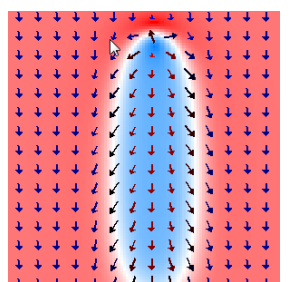

$\mathrm{H}=-1.0 \mathrm{~T}$

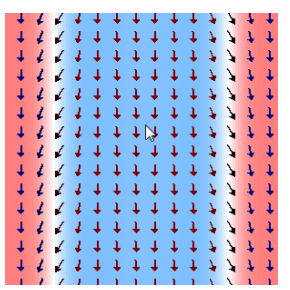

(c)

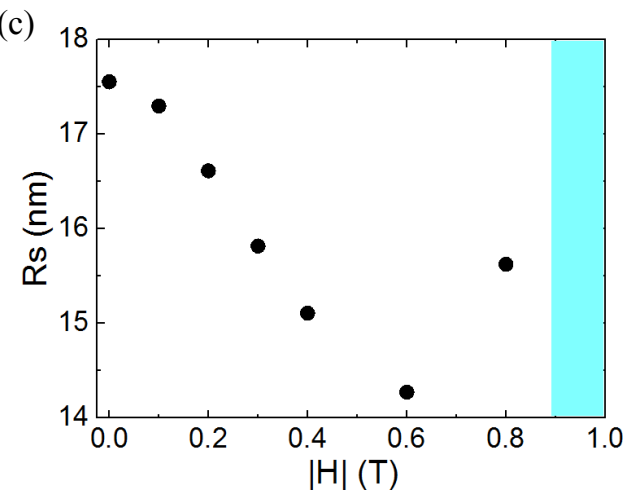

FIG. 6. (color online) (a) Spin configurations of the system as in-plane field decreases from 0 to $-1.0 \mathrm{~T}$. (b) $m_{y} \sim y$ for spins along the line $x=64 \mathrm{~nm}$ under $H=0$ (red line) and $H=-0.3 \mathrm{~T}$ (black line). The insets show the comparison of the skyrmion wall width with and without applied fields for cyan region and pink region, respectively. (c) Skyrmion size as a function of in-plane field strength. The skyrmion becomes unstable for $H>0.9 \mathrm{~T}$ (cyan region). Here the skyrmion size $(R)$ is defined by first counting the number of spins with $m_{z}>0(N)$, and then solving $R$ from the algebraic equation $\pi R^{2}=N d^{2}$, where $d$ is the mesh size.

be interpreted as magnon flow in the system ${ }^{19}$. For steady skyrmion motion, the translational symmetry of the skyrmion structures gives $\mathbf{m}_{s}\left(\mathbf{r}_{c}\right)=\mathbf{m}_{s}\left(\mathbf{r}_{c}-\mathbf{v} t\right)$, where $\mathbf{v}$ is skyrmion velocity and $\mathbf{r}_{c}$ is the short time average of skyrmion position, such that $\partial_{t} \mathbf{m}_{s}=-\mathbf{v} \cdot \nabla \mathbf{m}_{s}$. Performing the operation, $\int \mathbf{m}_{s} \cdot\left(\nabla \mathbf{m}_{s} \times\right.$ Eq. (7)), we obtain the generalized Thiele equation,

$$
\mathbf{F}+\mathbf{G} \times \mathbf{v}+\alpha \mathbf{D} \cdot \mathbf{v}=0,
$$

where $\mathbf{G}=4 \pi Q \mathbf{e}_{z}$ is the gyrovector of the skyrmion with $Q= \pm 1$ the topological charge of skyrmion, $D_{i j}=\int \partial_{i} \mathbf{m}$. $\partial_{j} \mathbf{m} d S$ is dissipation tensor, and $\mathbf{F}=-\int\left\langle\mathbf{m}_{\mathrm{f}} \times \mathbf{H}_{\mathrm{f}}\right\rangle$. $\left(\mathbf{m}_{s} \times \nabla \mathbf{m}_{s}\right) d x d y$ is the driven force coming from the magnon flow. The skyrmion velocity can be solved as

$$
v_{i}=-\frac{\alpha D F_{i}+4 \pi Q \epsilon_{3 i j} F_{j}}{(4 \pi Q)^{2}+\alpha^{2} D^{2}}
$$

where $i, j=1,2,3$ corresponds to $x, y, z$ coordinates. Alternatively, the dynamic equation Eq. (8) can be written as

$$
\mathbf{G} \times\left(\mathbf{v}-\mathbf{j}^{(m)}\right)+\alpha \mathbf{D} \cdot \mathbf{v}=0,
$$

where the magnon current $\mathbf{j}^{(m)}$ is defined as $\mathbf{F}=-\mathbf{G} \times$ $\mathbf{j}^{(m)}$. This form of Thiele equation is adopted in the main text.

For an arbitrary magnetic structure $\left(\mathbf{m}_{s}\right)$, the spin wave excitation can be written as $\mathbf{m}_{\mathrm{f}}=\left(m_{\theta} \mathbf{e}_{\theta}+\right.$ $\left.m_{\varphi} \mathbf{e}_{\varphi}\right) \mathbf{e}^{i \omega t}$, where $\mathbf{e}_{\varphi}$ and $\mathbf{e}_{\theta}$ are determined by the local magnetization direction $\mathbf{e}_{r} \equiv \mathbf{m}_{s}$. Then the effective fields due to exchange interaction, anisotropy term, Dzyaloshinskii-Moriya interaction term (DMI) and Zeeman term read, 


$$
\begin{aligned}
& \mathbf{H}_{\mathrm{f}, \mathrm{ex}}=2 A \nabla^{2} \mathbf{m}_{f}=2 A\left[-m_{\theta} \nabla^{2} \theta-\nabla m_{\theta} \cdot \nabla \theta\right] \mathbf{e}_{r}+2 A\left[\nabla^{2} m_{\theta}-m_{\theta}(\nabla \theta)^{2}\right] \mathbf{e}_{\theta}+2 A \nabla^{2} m_{\varphi} \mathbf{e}_{\varphi} \\
& \mathbf{H}_{\mathrm{f}, \mathrm{an}}=2 K m_{f, z} \mathbf{e}_{z}=-2 K m_{\theta} \sin \theta\left(\cos \theta \mathbf{e}_{r}-\sin \theta \mathbf{e}_{\theta}\right) \\
& \mathbf{H}_{\mathrm{f}, \mathrm{DM}}=D\left[\nabla \cdot \mathbf{m e}_{z}-\nabla m_{z}\right]=D \partial_{y}\left(m_{\theta} \mathbf{e}_{\theta}+m_{\varphi} \mathbf{e}_{\varphi}\right)_{y} e_{z}-\partial_{y}\left(m_{\theta} \mathbf{e}_{\theta}+m_{\varphi} \mathbf{e}_{\varphi}\right)_{z} \mathbf{e}_{y} \\
& \mathbf{H}_{\mathrm{f}, \mathrm{ze}}=0
\end{aligned}
$$

where we have assumed that the azimuthal angle $\phi$ is space independent, i.e. $\partial_{y} \varphi=0$. Then the exchange contribution to the driven force can be written as

$$
F_{\mathrm{ex}, \mathrm{y}}=-\int\left\langle\mathbf{m}_{\mathrm{f}} \times \mathbf{H}_{\mathrm{f}, \mathrm{ex}}\right\rangle \cdot\left(\mathbf{m}_{s} \times \partial_{y} \mathbf{m}_{s}\right)=-2 A \int \partial_{y} \theta m_{\theta}\left[m_{\theta} \nabla^{2} \theta+\nabla m_{\theta} \cdot \nabla \theta\right] d y
$$

where

$$
\begin{aligned}
\mathbf{m}_{s} \times \partial_{y} \mathbf{m}_{s} & =\partial_{y} \theta\left(-\sin \varphi e_{x}+\cos \varphi e_{y}\right) \\
\left\langle\mathbf{m}_{\mathrm{f}} \times \mathbf{H}_{\mathrm{f}, \mathrm{ex}}\right\rangle_{x} & =2 A\left[\sin \varphi m_{\theta}+\cos \theta \cos \varphi m_{\varphi}\right]\left[-m_{\theta} \nabla^{2} \theta-\nabla m_{\theta} \cdot \nabla \theta\right] \\
& +2 A \sin \theta \cos \theta \varphi m_{\theta} \nabla^{2} m_{\varphi}-2 A \sin \theta \cos \varphi m_{\varphi}\left[\nabla^{2} m_{\theta}-m_{\theta}(\nabla \theta)^{2}\right] \\
\left\langle\mathbf{m}_{\mathrm{f}} \times \mathbf{H}_{\mathrm{f}, \mathrm{ex}}\right\rangle_{y} & =2 A\left[-\cos \varphi m_{\theta}+\cos \theta \sin \varphi m_{\varphi}\right]\left[-m_{\theta} \nabla^{2} \theta-\nabla m_{\theta} \cdot \nabla \theta\right] \\
& +2 A \sin \theta \sin \theta \varphi m_{\theta} \nabla^{2} m_{\varphi}-2 A \sin \theta \sin \varphi m_{\varphi}\left[\nabla^{2} m_{\theta}-m_{\theta}(\nabla \theta)^{2}\right]
\end{aligned}
$$

Similarly, we can derive the contribution to $F_{y}$ from the anisotropy, DM interaction and Zeeman field as

$$
\begin{aligned}
& F_{\mathrm{an}, \mathrm{y}}=-2 K \int \partial_{y} \theta \sin \theta \cos \theta m_{\theta}^{2} d y \\
& F_{\mathrm{DM}, \mathrm{y}}=\frac{D}{2} \int \partial_{y} \theta \partial_{y} m_{\theta}^{2} d y \\
& F_{\mathrm{ze}, \mathrm{y}}=0 .
\end{aligned}
$$

It has been shown that the spin orientation in the radial direction of a skyrmion can be well described by a 360 domain wall ${ }^{40}$ in the form,

$$
\theta(r)=2 \arctan \left[\frac{\sinh (R / \Delta)}{\sinh (r / \Delta)}\right]
$$

where $R$ is skyrmion radius and $w$ is skyrmion wall width. Given $R \gg w$, this profile can be approximated as

$$
\theta(r)=-2 \arctan \left[\exp \frac{r-R}{\Delta}\right]
$$

which is the Walker profile for a $180^{\circ}$ domain wall, which will be used to further simplify the driven force. The driven force in the y-direction becomes

$$
\begin{aligned}
& F_{\text {ex }, \mathrm{y}}=-\frac{2 A}{\Delta^{3}} \int \sin ^{2} \theta m_{\theta}\left(\cos \theta m_{\theta}+\Delta \partial_{y} m_{\theta}\right) d y \\
& F_{\text {an }, \mathrm{y}}=-\frac{2 K}{\Delta} \int \sin ^{2} \theta \cos \theta m_{\theta}^{2} d y \\
& F_{\mathrm{DM}, \mathrm{y}}=\frac{D}{2 \Delta} \int \sin \theta \partial_{y} m_{\theta}^{2} d y \\
& F_{\mathrm{ze}, \mathrm{y}}=0
\end{aligned}
$$

where we have used the relations $\partial_{y} \theta=\sin \theta / \Delta, \partial_{y y} \theta=$ $\sin \theta \cos \theta / \Delta^{2}$ that is true for Walker profile of a magnetic structure.

In summary, the total force is

$$
F_{y}=-\frac{2 A}{\Delta^{3}} \int \sin ^{2} \theta m_{\theta}\left(\cos \theta m_{\theta}+\Delta \partial_{y} m_{\theta}\right) d y-\frac{2 K}{\Delta} \int \sin ^{2} \theta \cos \theta m_{\theta}^{2} d y+\frac{D}{2 \Delta} \int \sin \theta \partial_{y} m_{\theta}^{2} d y
$$

For a skyrmion with rotational symmetry, the spin wave excitation of the symmetric skyrmion wall $(H=0$ 

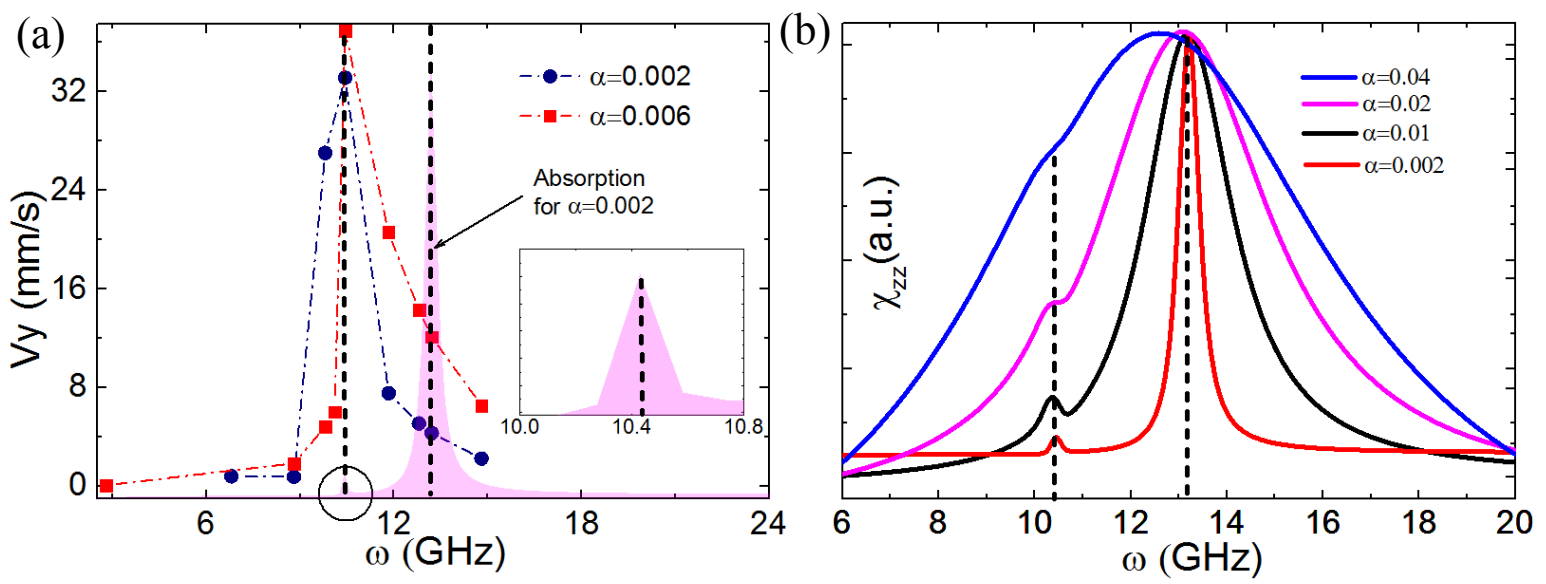

FIG. 7. (color online) (a) Skyrmion velocity as a function of electric field frequency for $\alpha=0.002$ (blue dots) and 0.006 (red squares), respectively. (b) Absorption spectrum of the magnetic system for $\alpha=0.04$ (blue line), 0.02 (pink color), 0.01 (black line), 0.002 (red line), respectively. The dashed lines refer to the positions of resonance peaks
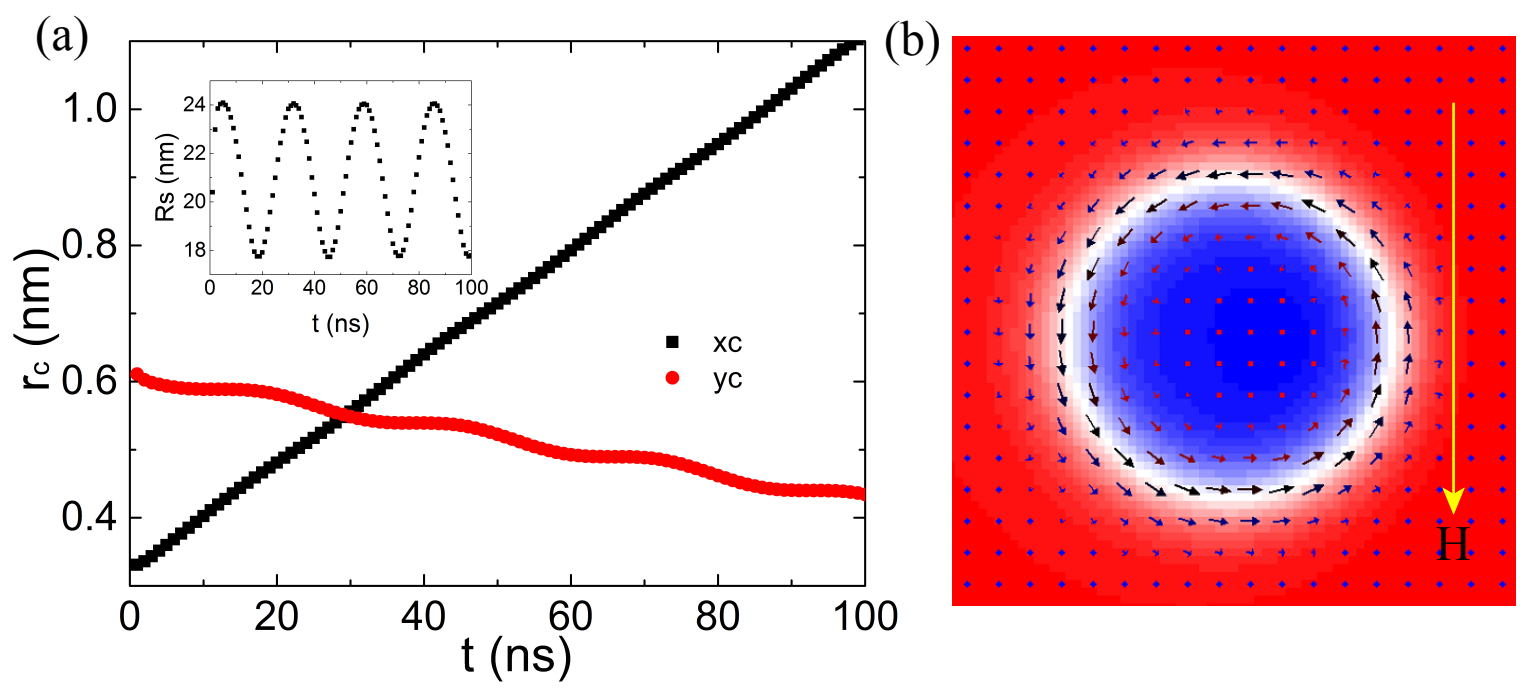

FIG. 8. (color online) (a) Bloch skyrmion position as a function of time by periodically tuning the anisotropy constant $K=$ $K_{u}+\Delta K \sin (\omega t)$. The parameters are $A=10^{-11} \mathrm{~J} / \mathrm{m}$, The bulk DMI strength $D=3 \mathrm{~mJ} / \mathrm{m}^{2}, K u=11.57 \times 10^{5} \mathrm{~J} / \mathrm{m}^{3}, \Delta K=$ $0.1 \times 10^{5} \mathrm{~J} / \mathrm{m}^{3}, \alpha=0.02, \omega=12.8 \mathrm{GHz}, H=-0.1 \mathrm{~T}$. The sampling rate is $1 \mathrm{frame} / \mathrm{ns}$. The inset shows the skyrmion radius as a function of time. (b) A static skyrmion profile. $H=-0.1 \mathrm{~T}$, the bulk DMI strength $D=3 \mathrm{~mJ} / \mathrm{m}^{2}$.

T in Fig. 6a) is also symmetric, hence $F_{y}=0$. For an asymmetric skyrmion, the spin wave excitation becomes asymmetric, where the narrower skyrmion wall (smaller $\Delta$ ) emit spin waves more intensively than the wider skyrmion wall (larger $\Delta$ ) as shown in Fig. 6b. Hence a net driven force in the asymmetric direction $(y)$ become non-zero. Moreover, due to the skyrmion Hall effect, the motion of skyrmion along the $y$ direction will induce a skyrmion motion along the $x$ direction and consequently deforms the skyrmion in the $x$ direction. As a result, a finite $F_{x}$ exist.

\section{APPENDIX C: SKYRMION VELOCITY IN THE LOW DAMPING REGIME}

Figure 7 a shows the skyrmion velocity as a function of field frequency for $\alpha=0.002$ (blue dots) and 0.006 (red squares), respectively. The position of maximum velocity shifts to $10.4 \mathrm{GHz}$, where a small absorption peak is identified. This suggests that the new mode makes significant contribution to the skyrmion velocity in the low damping regime. In larger damping regime, the role of this mode is dominated by the major mode around 13 $\mathrm{GHz}$ as shown in Fig. 7b. 

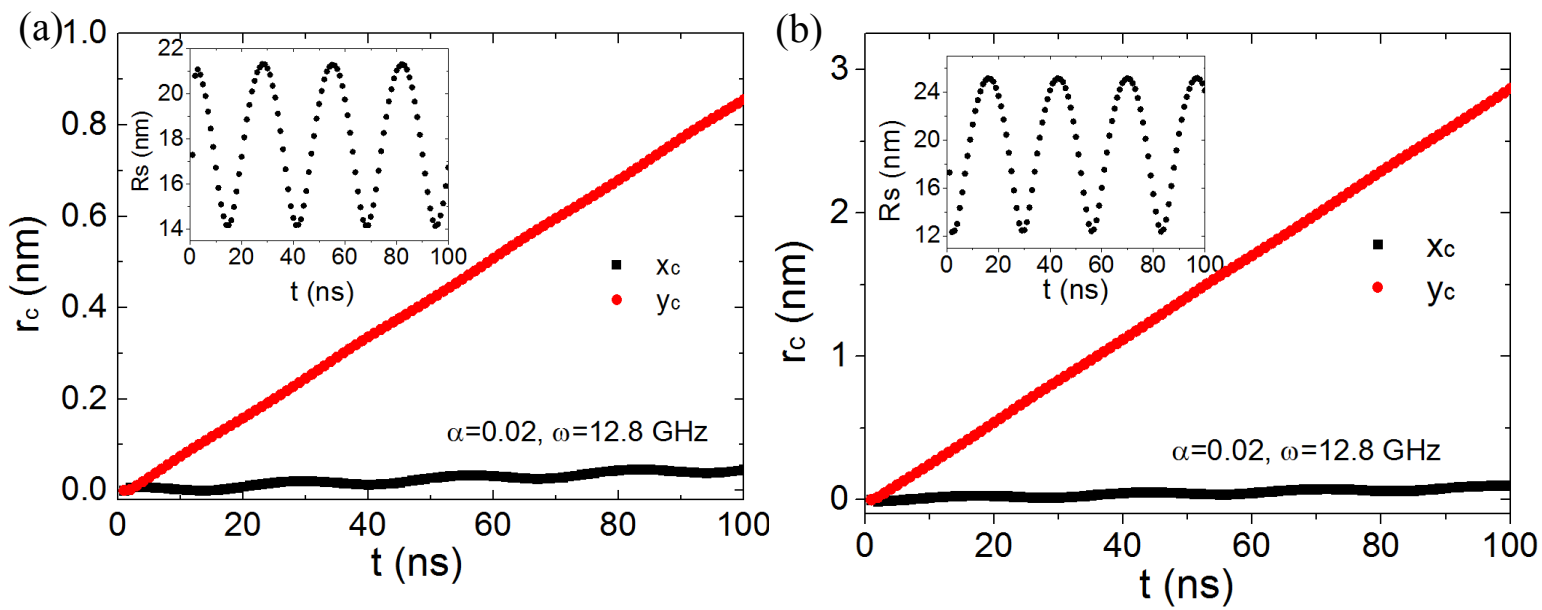

FIG. 9. (color online) Skyrmion position as a function of time by periodically tuning the exchange constant $A=A_{0}+$ $0.01 A_{0} \sin (\omega t)$ (a) and $D=D_{0}+0.01 D_{0} \sin (\omega t)(\mathrm{b})$, respectively. The inset shows the skyrmion radius as a function of time. The parameters are $A_{0}=10^{-11} \mathrm{~J} / \mathrm{m}, D_{0}=3 \mathrm{~mJ} / \mathrm{m}^{2}, K_{u}=11.57 \times 10^{5} \mathrm{~J} / \mathrm{m}^{3} . H=0.1 \mathrm{~T}$. The sampling rate is 1 frame/ns.

\section{APPENDIX D: BLOCH SKYRMION PROPAGATION DRIVEN BY PARAMETRIC PUMPING}

In this section, we show that Bloch skyrmion can also be driven to move under the periodical oscillation of magnetic parameters. Figure 8a shows the skyrmion position as a function of time for a Bloch skyrmion under the influence of the in-plane field $H_{y}=-0.1 \mathrm{~T}$ and the periodic pumping of magnetic anisotropy. The inset shows the oscillation of skyrmion radius. Figure $8 \mathrm{~b}$ shows the static profile of an asymmetric Bloch skyrmion.

\section{APPENDIX E: SKYRMION PROPAGATION BY OSCILLATING EXCHANGE STIFFNESS AND DMI STRENGTH}

In this section, we show two examples of moving skyrmions by periodically changing exchange stiffness and DMI. As shown in Fig. 9, the skyrmion obtains a finite speed of $8.5 \mathrm{~mm} / \mathrm{s}$ and $30 \mathrm{~mm} / \mathrm{s}$ by periodically tuning the exchange stiffness and DMI strength by only $1 \%$ percent while the skyrmion size oscillates during the propagation, which is similar to the case by tuning the anisotropy.
* Electronic address: yuanhy@sustc.edu.cn

$\dagger$ Electronic address: yung@sustc.edu.cn

¥ Electronic address: phxwan@ust.hk

1 A. N. Bogdanov and U. K. Rößler, Phys. Rev. Lett. 87, 037203 (2001).

2 U. K. Rößler, A. N. Bogdanov, and C. Pfleiderer, Nature 442, 797 (2006).

3 S. Mühlbauer, B. Binz, F. Jonietz, C. Pfleiderer, A. Rosch, A. Neubauer, R. Georgii, P. Böni1, Science 323, 915 (2009).

4 X. Z. Yu, Y. Onose,, N. Kanazawa, J. H. Park, J. H. Han, Y. Matsui, N. Nagaosa, and Y. Tokura, Nature 465, 901 (2010).

5 X. Z. Yu, N. Kanazawa, Y. Onose, K. Kimoto, W. Z. Zhang, S. Ishiwata, Y. Matsui, and Y. Tokura Nat. Mater. 10, 106 (2011).

${ }^{6}$ S. Woo, K. Litzius, B. Krüger, M.-Y. Im, L. Caretta, K. Richter, M. Mann, A. Krone, R. M. Reeve, M. Weigand, P. Agrawal, I. Lemesh, M.-A. Mawass, P. Fischer, M. Kläui, and G. S. D. Beach, Nat. Mater. 15, 501 (2016).

7 H. Y. Yuan and X. R. Wang, Sci. Rep. 6, 22638 (2016).

8 H. Y. Yuan, O. Gomonay, and Mathias Kläui, Phys. Rev. B 96, 134415 (2017).
9 A. P. Malozemoff and J. C. Slonczewski, Magnetic domain walls in bubble materials (Academic Press, 1979).

10 H. Y. Yuan and X. R. Wang, Phys. Rev. B 92, 054419 (2015).

11 A. Siemens, Y. Zhang, J. Hagemeister, E. Y. Vedmedenko and R. Wisendanger, New J. Phys. 18, 045021 (2016).

12 J. Iwasaki, M. Mochizuki, and N. Nagaosa, Nat. Commun. 4, 1463 (2013).

13 Y. Zhou and M. Ezawa, Nat. Commun. 5, 4652 (2014).

14 K. Litzius, I. Lemesh, B. Krüger, P. Bassirian, L. Caretta, K. Richter, F. Büttner, K. Sato, O. A. Tretiakov, J. Förster, R. M. Reeve1, M. Weigand, I. Bykova, H. Stoll, G. Schtz, G. S. D. Beach, and M. Kläui, Nat. Phys. 13, 170 (2017).

15 J. Iwasaki, A. J. Beekman, and N. Nagaosa, Phys. Rev. B 89, 064412 (2014).

16 C. Schutte and M. Garst, Phys. Rev. B 90, 094423 (2014).

17 W. Wang, M. Beg, B. Zhang, W. Kuch, and H. Fangohr, Phys. Rev. B 92, 020403 (R) (2015).

18 W. Yang, H. Yang, Y. Cao, and P. Yan, Optical Express 26, 8778 (2018).

19 L. Kong and J. Zang, Phys. Rev. Lett. 111, 067203 (2013). 
20 S.-Z. Lin, C. D. Batista, C. Reichhardt, and A. Saxena, Phys. Rev. Lett. 112, 187203 (2014).

21 M. Mochizuki, X. Z. Yu, S. Seki, N. Kanazawa, W. Koshibae, J. Zang, M. Mostovoy, Y. Tokura, and N. Nagaosa, Nat. Mater. 13, 241 (2014).

${ }^{22}$ W. Jiang, X. Zhang, G. Yu, W. Zhang, X. Wang, M. B. Jungfleisch, J. E. Pearson, X. Cheng, O. Heinonen, K. L. Wang, Y. Zhou, A. Hoffmann, and S. G. E. te Velthuis, Nat. Phys. 13, 162 (2017).

${ }^{23}$ P. Yan, X. S. Wang, and X. R. Wang, Phys. Rev. Lett. 107, 177207 (2011).

${ }^{24}$ F. Matsukura, Y. Tokura, and H. Ohno, Nat. Nanotech. 10, 209 (2015) and the references therein.

${ }^{25}$ H. Ohno, D. Chiba, F. Matsukura, T. Omiya, E. Abe, T. Dietl, Y. Ohno, and K. Ohtani, Nature 408, 944 (2000).

${ }^{26}$ F. Ando, H. Kakizakai, T. Koyama, K. Yamada, M. Kawaguchi, S. Kim, K.-J. Kim, T. Moriyama, D. Chiba, and T. Ono, Appl. Phys. Lett. 109, 022401 (2016).

27 T. Dohi, S. Kanai, A. Okada, F. Matsukura, and H. Ohno, AIP Advances 6, 075017 (2016).

28 H. Yang, O. Boulle, V. Cros, A. Fert, and M. Chshiev, arXiv:1603.01847v2.

29 M. Weisheit, S. Fähler, A. Marty, Y. Souche, C. Poinsignon, and D. Givord, Science 315, 349 (2007).

30 T. Maruyama, Y. Shiota1, T. Nozaki, K. Ohta, N. Toda, M. Mizuguchi, A. A. Tulapurkar, T. Shinjo, M. Shiraishi, S. Mizukami, Y. Ando, and Y. Suzuki, Nat. Nanotech. 4, 158 (2009).

31 D. Lebeugle, A. Mougin, M. Viret, D. Colson, and L. Ranno, Phys. Rev. Lett. 103, 257601 (2009).

32 J. T. Heron, M. Trassin, K. Ashraf, M. Gajek, Q. He, S. Y. Yang, D. E. Nikonov, Y. H. Chu, S. Salahuddin, and R. Ramesh, Phys. Rev. Lett. 107, 217202 (2011).

33 A. J. Schellekens, A. van den Brink, J. H. Franken, H. J. M. Swagten, and B. Koopmans, Nat. Commun. 3, 847(2012).

${ }^{34}$ D. Chiba, M. Kawaguchi, Si Fukami, N. Ishiwata, K. Shimamura, K. Kobayashi, and T. Ono, Nat. Commun. 3, 888 (2012).

35 K. J. A. Franke, B. VandeWiele, Y. Shirahata, S. J. Hamalainen, T. Taniyama, and S. vanDijken, Phys. Rev. X 5, 011010 (2015).

36 I. E. Dzyaloshinskii, Sov. Phys. JETP 5, 1259 (1957).

37 T. Moriya, Phys. Rev. 120, 91 (1960).

38 P. Upadhyaya, G. Yu, P. K. Amiri, and K. L. Wang, Phys. Rev. B 92, 134411 (2015).

39 A. Vansteenkiste, J. Leliaert, M. Dvornik, M. Helsen, F. Garcia- Sanchez, and F. B. V. Waeyenberge, AIP Adv. 4, 107133 (2014).

${ }^{40}$ X. S. Wang, H. Y. Yuan, and X. R. Wang, Commun. Phys. 1, 31 (2018).

41 S. Rohart and A. Thiaville, Phys. Rev. B 88, 184422 (2013).

42 V. P. Kravchuk, D. D. Sheka, U. K. Rossler, J. vandenBrink, and Y. Gaididei, Phys. Rev. B 97, 064403 (2018).

43 Y. Zhang, X. S. Wang, H. Y. Yuan, S. S. Kang, H. W. Zhang, and X. R. Wang, J. Phys.: Condens. Matter 29, 095806 (2017).

44 M. Mochizuki, Phys. Rev. Lett. 108, 017601 (2012).

${ }^{45}$ Y. Onose, Y. Okamura, S. Seki, S. Ishiwata, and Y. Tokura, Phys. Rev. Lett. 109, 037603 (2012).

46 J.-V. Kim, F. Garcia-Sanchez, J. Sampaio, C. MoreauLuchaire, V. Cros, and A. Fert, Phys. Rev. B 90, 064410 (2014).
47 X. S. Wang, P. Yan, Y. H. Shen, G. E. W. Bauer, and X. R. Wang, Phys. Rev. Lett. 109, 167209 (2012); X. S. Wang and X. R. Wang, Phys. Rev. B 90, 184415 (2014)

48 A. Fasano and S. Marmi, Analytical Mechanics, Oxford University Press. (Oxford, New York, 2002).

49 R. Schirhagl, K. Chang, M. Loretz, and C. L. Degen, Annu. Rev. Phys. Chem. 65, 83 (2014).

50 J. J. Sakura and J. Napolitano, Modern Quantum Mechanics, 2nd edition, (Addison-Wesley, 2011, Boston, Columbus et al.)) 